\title{
Experimental observation of Shapiro-steps in colloidal monolayers driven across time-dependent substrate potentials
}

\author{
T. Brazda, C. July and C. Bechinger (D)*
}

\begin{abstract}
We experimentally study the motion of a colloidal monolayer which is driven across a commensurate substrate potential whose amplitude is periodically modulated in time. In addition to a significant reduction of the static friction force compared to an unmodulated substrate, we observe a Shapiro step structure in the force dependence of the mean particle velocity which is explained by the dynamical mode locking between the particle motion and the substrate modulation. In this regime, the entire crystal moves in a stick-slip fashion similar to what is observed when a single point contact is driven across a periodic surface. Contrary to numerical simulations, where typically a large number of Shapiro steps is found, only a single step is observed in our experiments. This is explained by the formation of kinks which weaken the synchronization between adjacent particles.
\end{abstract}

\section{Introduction}

Although friction is omnipresent in our daily life and systematic studies date back more than 300 years, many aspects of tribology remain poorly understood. Even when considering one of the simplest examples, i.e. two atomically flat surfaces in close contact and which are sliding across each other, a rather complex particle dynamics occurs. In addition to the well-known stick-slip motion, ${ }^{1-3}$ the Frenkel-Kontorova (FK) model predicts the formation of topological defects such as kinks and antikinks which provide an efficient mechanism for mass transport across the interface and are thus crucial for a quantitative understanding of the friction coefficient. ${ }^{4-9}$ Recently, experiments with colloidal monolayers allowed detailed insights into the particle motion relative to the substrate potential and thus revealed direct evidence for the motion and lateral structure of kinks and antikinks. ${ }^{10}$ Compared to atomic systems where e.g. the lattice spacing and the elasticity of the two interacting bodies are determined by the specific choice of the materials, colloidal model systems offer a higher flexibility regarding such material parameters. In general, colloidal systems are distinguished by ultralow elastic constants ${ }^{11}$ (about 9 orders of magnitude smaller compared to solids). Accordingly, their response to local strain-fields is much higher which allows to resolve minute changes in the structural properties of such systems.

In contrast to previous measurements where the frictional properties of a colloidal monolayer on static substrate potentials

12. Physikalisches Institut, Universitat Stuttgart, Pfaffenwaldring 57 , 70569 Stuttgart, Germany. E mail: c.bechinger@physik.uni stuttgart.de was studied, ${ }^{10,12}$ here we consider the situation where the substrate potential amplitude periodically oscillates in time. Under such conditions, numerical simulations predict the occurrence of Shapiro steps in the mean particle velocity which results from the dynamical phase locking between the particle motion and the substrate oscillation. ${ }^{13,14}$

\section{Experimental}

In our experiments, we have used negatively charged polystyrene spheres with radius $R=1.95 \mu \mathrm{m}$ and about $3 \%$ polydispersity which are suspended in an aqueous solvent. The colloidal particles are contained in a sample cell composed of two fused silica slides with a plate separation of $200 \mu \mathrm{m}$. After sedimentation and at sufficiently high particle densities, the particles assemble in an extended (several square millimeters) two-dimensional hexagonal crystal. Due to the interplay of gravitational and electrostatic forces the monolayer is confined at the bottom of the sample cell. A vertically incident laser beam exerts an additional light pressure and thus ensures that vertical particle fluctuations are reduced to about $2 \%$ of the particle diameter, i.e. that the system remains two-dimensional even in the presence of external driving. ${ }^{10}$ To enhance the electrostatic particle interaction, ions have been largely removed from the suspension by a closed deionizing circuit. ${ }^{15}$ Depending on the degree of deionization, this leads to Debye screening lengths up to $\kappa^{-1} \approx 180 \mathrm{~nm}$ which can be maintained over several hours. This value has been determined by the comparison between measured and numerically computed pair correlation functions. Substrate potentials were created by a 
polarized beam of a frequency-doubled solid state laser $(\lambda=532 \mathrm{~nm})$ which is split into three beams of identical intensity and polarization. These beams are directed from above into the sample cell where they create a hexagonal lattice. Due to optical gradient forces, this optical landscape acts as substrate potential for the colloidal particles. Because the substrate strength $U$ is proportional to the incident light intensity, it can be adjusted continuously. ${ }^{16}$ To achieve commensurate conditions between the colloidal crystal and the substrate potential, the optical lattice spacing $s$ (being determined by the angle of incidence of the interfering laser beams) was adjusted to be identical to the mean particle spacing $a$. In the experiments reported here, we have chosen $s=a=6.1 \mu \mathrm{m}$. Using an acousto-optical modulator (AOM), we vary the intensity of the interfering laser beams which leads to a time-dependent and spatially periodic substrate potential $U(x, y, t)=\left[U_{0}+\Delta U \cos \left(2 \pi t / T_{\Omega}\right)\right] f(x, y)$ where

$$
f(x, y)=\left(\frac{2}{9}\right)\left[\frac{3}{2}+\cos \left(\frac{4 \pi y}{\sqrt{ } 3 s}\right)+2 \cos \left(\frac{2 \pi y}{\sqrt{ } 3 s}\right) \cos \left(\frac{2 \pi x}{s}\right)\right]
$$

describes the spatial $(x, y)$ modulation of the intensity pattern. As a function of time $t$, the potential oscillates with period $T_{\Omega}$ between $U=U_{0} \pm \Delta U$. In our experiments, $U_{0}$ has been chosen to be larger than $\Delta U$ which renders the spatial position of the potential maxima and minima to be constant.

Lateral driving forces to the colloidal monolayer were applied by moving the entire sample cell along one of the symmetry directions of the crystal by a piezo stage with velocity $u$. Accordingly, each colloid is laterally driven with the Stokes force $F=\eta_{\text {eff }} u$ where the effective viscosity $\eta_{\text {eff }}$ has been determined from the measured short-time particle diffusion coefficient. Its value is about $30 \%$ higher than the bulk viscosity of water due to hydrodynamic interactions between the particles and the substrate. ${ }^{17}$ Particle positions were recorded with a CCD camera with a frame rate of $3 \mathrm{~s}^{-1}$ and stored for later data analysis. All experiments were performed at room temperature.

\section{Results}

Fig. 1a compares the time-averaged colloidal drift velocity $v_{\mathrm{d}}=\frac{1}{N} \sum_{i}^{N} v_{i}$ ( $N$ being the total number of particles) of a crystal on a static (open symbols) and a modulated (closed symbols) substrate potential. In agreement with previous experiments ${ }^{18,19}$ and simulations, ${ }^{20,21}$ we observe with increasing $F$ a transition from a pinned to a sliding monolayer. For the parameters used here $\left(U_{0}=5.6 k_{\mathrm{B}} T\right)$, depinning occurs at $F \cong 22 \mathrm{fN}$. Above this value, the monolayer partially depinnes due to the formation of kinks and antikinks and finally slides almost undistorted across the surface. ${ }^{10}$ For an oscillating substrate, the drift velocity curve looks qualitatively different. Exemplarily, we show the results for $T_{\Omega}=90 \mathrm{~s}$ and $\Delta U=21 k_{\mathrm{B}} T$ but qualitatively similar results have been also observed for other amplitudes and oscillation periods. In addition to a rather abrupt change from a pinned to a sliding state, a pronounced step-like structure is observed for $33<F<$ $47 \mathrm{fN}$. To yield comparable results for the depinning forces with

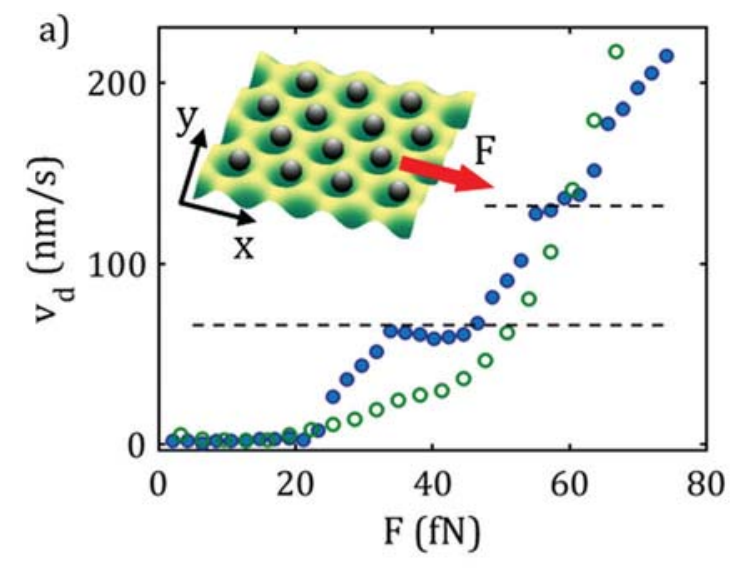

b)

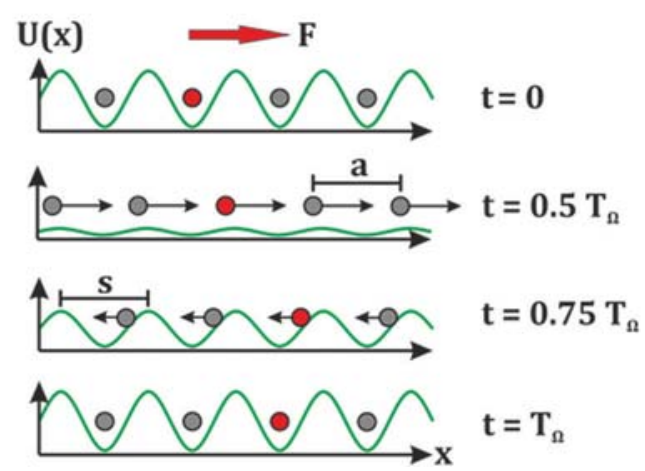

Fig. 1 (a) Mean particle drift velocity of a driven commensurate colloidal monolayer on a hexagonal substrate potential as a function of the lateral driving force $F$. Open and closed symbols correspond to a static $\left(U_{0}=5.6 k_{\mathrm{B}} T\right)$ and oscillating $\left(T_{\Omega}=90 \mathrm{~s}, U_{0}=23 k_{\mathrm{B}} T, \Delta U=21 k_{\mathrm{B}} T\right)$ substrate potential. Dashed lines show the positions where phase locking Shapiro steps are expected. Inset: Schematic drawing of a driven colloidal mono layer on a hexagonal substrate potential. (b) Explanation of the origin of the phase locking mechanism for a one dimensional systems of interacting particles in a periodic substrate potential.

the unmodulated situation, the mean substrate potential has been adjusted to $U_{0}=23 k_{\mathrm{B}} T$.

The observed step-like structure is in agreement with recent Brownian dynamics simulations on colloidal systems ${ }^{13,14}$ and originate from a dynamical phase locking between the particle motion and the oscillation of the substrate and is schematically explained in Fig. 1b. At $t=0 \mathrm{~s}$ where the substrate potential is largest, the colloids are strongly trapped at the potential minima. This leads to $v_{\mathrm{d}}=0 \mathrm{~nm} \mathrm{~s}^{-1}$. For $t=0.5 T_{\Omega}$ the potential barriers become minimal which facilitates their surmounting by colloids and enables the entire colloidal crystal to slide in the direction of $F$. With increasing time, potential barriers will increase again and particles relax back to the nearest potential minimum. When the relaxation time $\tau$ of particles within the substrate potential is much smaller than $T_{\Omega}$, after each oscillation period $t=n T_{\Omega}$ ( $n$ being an integer), the particles become again localized at potential minima (independent of their previous position within the potential well) but are shifted in the direction of $F$. In the example shown in Fig. 1b, the particles have laterally moved by one substrate lattice constant $s$ during a modulation cycle and the averaged particle drift velocity is $v_{\mathrm{d}}=n s / T_{\Omega}$. 
This dynamical phase locking leads to the Shapiro steps in the drift velocity. The position of the experimentally observed steps in Fig. 1a are in excellent agreement with this prediction (dashed horizontal lines). Contrary to numerical simulations, ${ }^{13}$ however, where a large number of equidistant Shapiro steps is observed, in our experiments Shapiro steps are hardly visible for $n>1$.

To understand, why Shapiro steps rapidly disappear with increasing $n$, we have studied the monolayer motion with single particle resolution. Fig. 2a shows the $x$-component (parallel to the driving force) of particle trajectories within a representative section of the sample. The data correspond to $F=43 \mathrm{fN}$ where a Shapiro plateau is experimentally observed ( $c f$. Fig. 1a). In agreement with the phase-locking mechanism explained above, all particles move in a synchronous and periodic fashion which leads to a particle displacement by one lattice constant after each oscillation period. Because the transition probability varies exponentially with the potential barrier proportional to $\exp \left(U / k_{\mathrm{B}} T\right)$, this explains why particle motion occurs only around times corresponding to odd multiples of $T_{\Omega} / 2$, where the potential barriers are smallest (see also Fig. 1b). Under our conditions, particle motion occurs when $U \leq 5 k_{\mathrm{B}} T$. Similar trajectories are observed over the entire range of the first Shapiro step (33 fN $<F<46 \mathrm{fN}$ ).

Fig. $2 \mathrm{~b}$ shows the corresponding data for $F=58 \mathrm{fN}$. At these driving forces the second Shapiro step should appear ( $c f$. Fig. 1a) and indeed the particle displacement during an oscillation period is equal to $2 \mathrm{~s}$ (inset of Fig. 2b). To show the collective particle motion, in Fig. 2c we have plotted particle trajectories over a larger spatial region (same parameters as in Fig. 2b). In contrast to Fig. 2a,

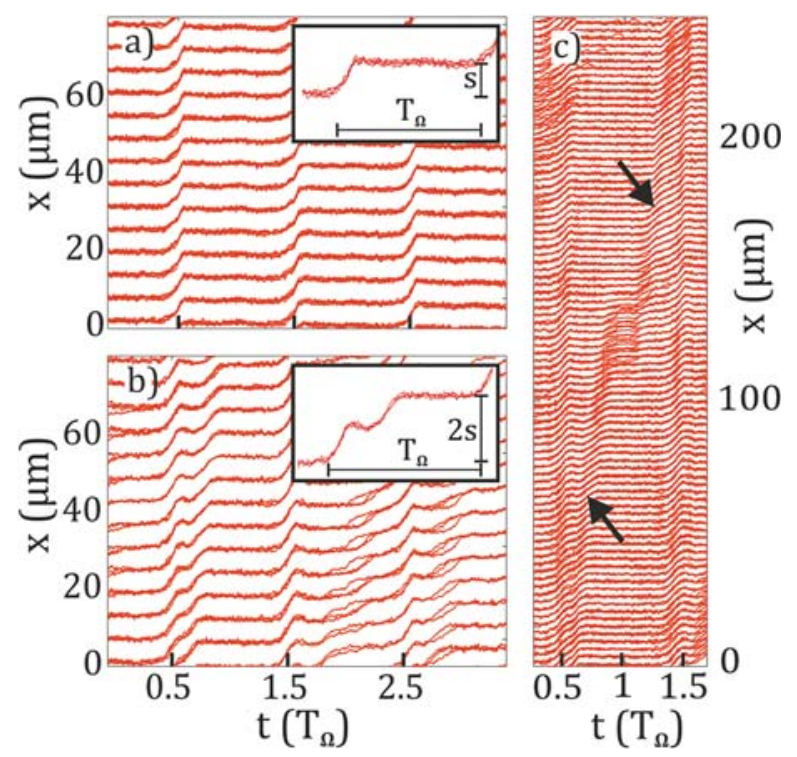

Fig. 2 Kink propagation within a colloidal monolayer. Solid lines correspond to the $x$ component of the particle trajectories parallel to the driving force on an oscillating substrate $\left(T_{\Omega}=90 \mathrm{~s}, U_{0}=23 k_{\mathrm{B}} T, \Delta U=21 k_{\mathrm{B}} T\right)$. (a) For $F_{\mathrm{d}}=40 \mathrm{fN}$ where phase locking occurs, particle move simultaneously. (b) For $F_{\mathrm{d}}=58 \mathrm{fN}$ particle motion is less synchronized which leads to the formation of kinks. (c) Extended view of the trajectories shown in (b). The black arrows mark moving kinks. where particle motion occurs only at odd multiples of $0.5 T_{\Omega}$, in Fig. 2c some colloids are also able to surmount rather large potential barriers (e.g. at $\left.t \approx 0.75 T_{\Omega}\right)$. Such unlocked particle motion leads to localized compression zones which propagate in the direction of the applied force (arrows in Fig. 2c). Similar features have been previously reported in colloidal crystals which are driven across static substrate potentials where they were identified as running kinks. ${ }^{9}$ Contrary to that, however, the kinks in Fig. 2c do not propagate with constant speed but their velocity can even drop to zero (frozen kinks) as seen in the center of Fig. 2c.

Local compressions within the monolayer can be quantified by measuring the probability distribution of the Voronoi cell area $P(A)$. Because the strength of the substrate potential varies over time, a time-dependence of $P(A)$ is expected. Fig. 3a shows $P(A)$ for $F=40 \mathrm{fN}$ where the first Shapiro plateau occurs and the particles move synchronously over the substrate. The blue and red data correspond to times, where the substrate potential is smallest $\left(U=2 k_{\mathrm{B}} T\right.$ at $\left.t=(n+1 / 2) T_{\Omega} ; n=0,1,2, \ldots\right)$ and largest $\left(U=45 k_{\mathrm{B}} T\right.$ at $\left.t=n T_{\Omega}\right)$, respectively. In both cases, $P(A)$ is well described by a Gaussian distribution whose half width becomes smaller with increasing $U$. This is due to the stronger particle localization at larger substrate potentials.

Fig. $3 \mathrm{~b}$ shows the corresponding data for $F=58 \mathrm{fN}$ where the second Shapiro plateau should occur ( $c f$. Fig. 2b). Similar to above, at times where the substrate potential is smallest (blue data), $P(A)$ follows a Gaussian distribution. However, when $U$ becomes maximal (red data), $P(A)$ becomes strongly asymmetric. In particular the shoulder towards smaller Voronoi cells suggests, that highly compressed regions form. This is in agreement with the creation of kinks already observed in Fig. 2b. To quantify the strain due to kinks, we first fitted a superposition of two Gaussians (solid line) to $P(A)$ in Fig. $3 \mathrm{~b}$. As a measure for the strain caused by kinks, we consider the area $A_{\mathrm{k}}$ which is defined by the integral under the left handed Gaussian up to the intersection point with the other Gaussian curve (shaded region in Fig. 3c).

In Fig. 4 we have plotted as open symbols $A_{\mathrm{k}} /\langle A\rangle$ as a function of the driving force. Here $\langle A\rangle$ corresponds to the total Voronoi cell area (i.e. the integral of the solid curve in Fig. 3c). For comparison, we have added the averaged particle drift velocity $v_{\mathrm{d}}$ as closed symbols ( $c f$. Fig. 1a). Even for very small driving forces $(F<22 \mathrm{fN})$, where $v_{\mathrm{d}} \approx 0$, a small elastic strain arises. This is because the probability for particle jumps over substrate potential barriers generally increases with increasing $F$. As a result, even below the static friction force which is characterized by an abrupt rise of $v_{\mathrm{d}}$, a small number (typically $4 \%$ ) of particles already cross the potential barrier which leads to a finite strain. When $F$ becomes stronger, the strain further increases until it significantly drops at driving forces where the first Shapiro plateau is observed. As already mentioned above, under such conditions synchronous particle motion occurs which strongly reduces the accumulation of elastic strain. Further increasing $F$ leads to a monotonic increase of $\left\langle A_{\mathrm{k}}\right\rangle$ even within the range where the second Shapiro step is expected (dashed line) and thus confirms the partially asynchronous trajectories as observed in Fig. $2 \mathrm{~b}$ and c. 


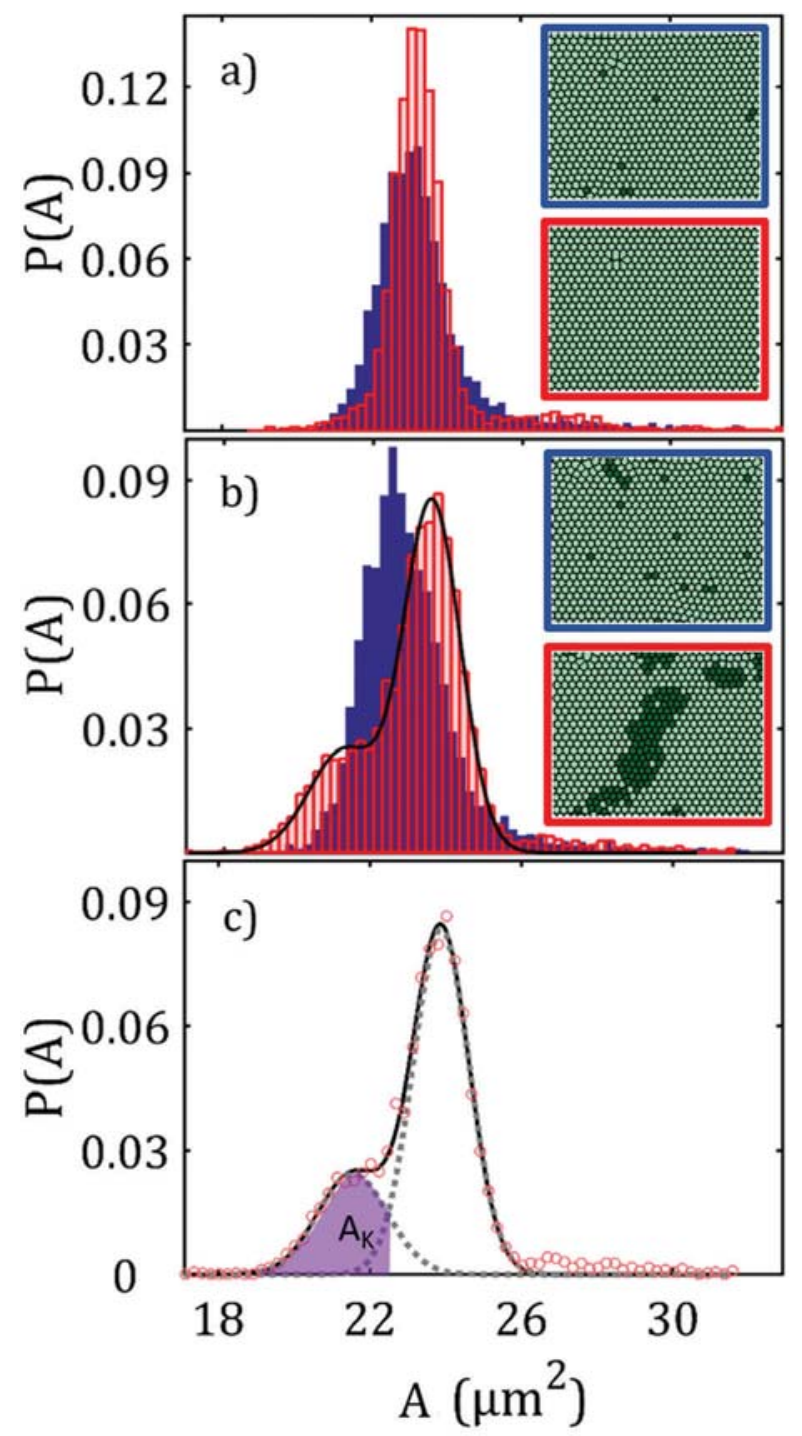

Fig. 3 Histogram of the Voronoi area distribution for times where the modulated substrate potential (same parameters as in Fig. 2) is smallest (blue), i.e. for $t=(n+1 / 2) T_{\Omega} ; n=0,1,2, \ldots$, and largest (red), i.e. for $t=n T_{\Omega}$. (a) $F=40 \mathrm{fN}$ and (b) $F=58 \mathrm{fN}$. Insets: Voronoi tessellation of the colloidal crystal, with large and small Voronoi cells shown in light and dark green, respectively. (c) Histogram of (b) for $U_{\max }=45 k_{\mathrm{B}} T$ (red circles) with bimodal Gaussian fit (solid line). The dotted lines show two Gaussians which sum up to the solid line. The shaded region corresponds to a measure for the total strain in the colloidal crystal. It is defined by the integral over the left hand Gaussian curve with the upper integration limit set by the intersection point between the two Gaussian curves.

In general the occurence of Shapiro steps increases with increasing inter-particle coupling. In contrast to numerical simulations, where the ratio of the colloidal lattice constant a and the Debye screening length $a / \kappa^{-1}$ was set to $2,{ }^{13}$ in our experiments this value is $\approx 30$. This explains why higher order Shapiro steps are not observed by us. In addition to the strength of the particle coupling, numerical studies suggest that the number of Shapiro steps should also depend on the substrate modulation time $T_{\Omega} \cdot{ }^{18,22-26}$ For example, when the relaxation time $\tau$ is much larger than $T_{\Omega}$, particles do not

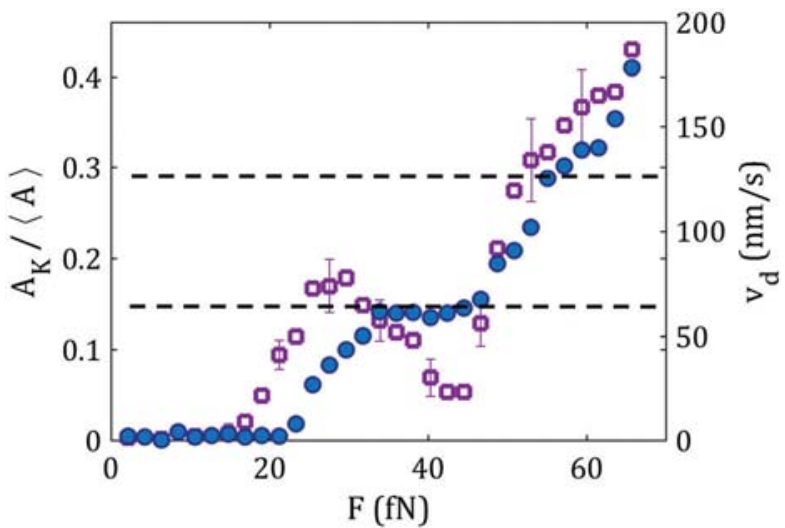

Fig. 4 Area of the kinks vs. $F_{\mathrm{d}}$ (open squares) in comparison to the average particle velocity (closed circles) $\left(T_{\Omega}=90 \mathrm{~s}, U_{0}=23 k_{\mathrm{B}} T, \Delta U=21 k_{\mathrm{B}} T\right)$. Dashed lines show the positions where phase locking Shapiro steps are expected.

fully relax to potential minima after each modulation cycle $\left(t=(n+1 / 2) T_{\Omega}\right)$. This results in a narrowing and eventually the disappearance of single Shapiro steps. Since $T_{\Omega}$ in our experiments can be only varied over a rather small range, however, such a dependence cannot be investigated with our setup.

\section{Conclusions}

In conclusion, we have experimentally demonstrated the dynamical phase locking of a colloidal monolayer which is driven across an oscillating periodic substrate potential. In contrast to stiff monolayers, where a large number of such steps has been predicted, only a single pronounced Shapiro step is found in our experiments. This is due to the formation of local density fluctuations (kinks) which weaken the synchronous particle motion and thus lead to the disappearance of higher order Shapiro steps.

\section{Acknowledgements}

We acknowledge Andrea Silva for the numerical calculation of pair correlation functions and Celia Lozano for her help with the artwork.

\section{Notes and references}

1 E. Gnecco, R. Bennewitz, T. Gyalog, C. Loppacher, M. Bammerlin, E. Meyer and H.-J. Güntherodt, Phys. Rev. Lett., 2000, 84, 1172.

2 C. Lee, Q. Li, W. Kalb, X.-Z. Liu, H. Berger, R. W. Carpick and J. Hone, Science, 2010, 328, 76-80.

3 G. Tomlinson, The London, Edinburgh, and Dublin philosophical magazine and journal of science, 1929, 7, 905-939.

4 S. Aubry and P.-Y. Le Daeron, Phys. D, 1983, 8, 381-422.

5 O. M. Braun and Y. Kivshar, The Frenkel-Kontorova model: concepts, methods, and applications, Springer Science \& Business Media, 2013.

6 O. M. Braun and Y. S. Kivshar, Phys. Rep., 1998, 306, 1-108. 7 L. M. Floría and J. J. Mazo, Adv. Phys., 1996, 45, 505-598. 
8 T. Kontorova and J. Frenkel, Zh. Eksp. Teor. Fiz., 1938, 8, 1340-1348.

9 C. Reichhardt and C. O. Reichhardt, Rep. Prog. Phys., 2016, 80, 026501.

10 T. Bohlein, J. Mikhael and C. Bechinger, Nat. Mater., 2012, 11, 126-130.

11 D. Frenkel, Phys. A, 2002, 313, 1-31.

12 T. Bohlein and C. Bechinger, Phys. Rev. Lett., 2012, 109, 058301.

13 A. Libal, C. Reichhardt, B. Janko and C. O. Reichhardt, Phys. Rev. Lett., 2006, 96, 188301.

14 S. V. P. Ticco, G. Fornasier, N. Manini, G. E. Santoro, E. Tosatti and A. Vanossi, J. Phys.: Condens. Matter, 2016, 28, 134006.

15 T. Palberg, W. Härtl, U. Wittig, H. Versmold, M. Würth and E. Simnacher, J. Phys. Chem., 1992, 96, 8180-8183.

16 J. Baumgartl, J. Dietrich, J. Dobnikar, C. Bechinger and H. H. von Grünberg, Soft Matter, 2008, 4, 2199-2206.

17 H. Brenner, Chem. Eng. Sci., 1961, 16, 242-251.
18 M. P. Juniper, A. V. Straube, R. Besseling, D. G. Aarts and R. P. Dullens, Nat. Commun., 2015, 6, 7187.

19 M. P. Juniper, U. Zimmermann, A. V. Straube, R. Besseling, D. G. Aarts, H. Löwen and R. P. Dullens, New J. Phys., 2017, 19, 013010.

20 J. Hasnain, S. Jungblut and C. Dellago, Soft Matter, 2013, 9, 5867-5873.

21 A. Vanossi, N. Manini and E. Tosatti, Proc. Natl. Acad. Sci. U. S. A., 2012, 109, 16429-16433.

22 B. Hu and J. Tekić, Appl. Phys. Lett., 2007, 90, 102119.

23 B. Hu and J. Tekić, Phys. Rev. E: Stat., Nonlinear, Soft Matter Phys., 2007, 75, 056608.

24 C. Reichhardt, R. T. Scalettar, G. T. Zimányi and N. GrønbechJensen, Phys. Rev. B: Condens. Matter Mater. Phys., 2000, 61, R11914.

25 Y. Yang, W.-S. Duan, L. Yang, J.-M. Chen and M.-M. Lin, Europhys. Lett., 2011, 93, 16001.

26 P. Romanczuk, F. Müller and L. Schimansky-Geier, Phys. Rev. E: Stat., Nonlinear, Soft Matter Phys., 2010, 81, 061120. 